\title{
The space of states of quantum gravity in terms of loops and extended loops: some remarks
}

\author{
Cayetano Di Bartolo ${ }^{1}$, Rodolfo Gambini ${ }^{2}$, Jorge Griego ${ }^{2}$ and Jorge Pullin ${ }^{3}$ \\ 1. Departamento de Física, Universidad Simón Bolivar, Caracas, Venezuela \\ 2. Instituto de Física, Facultad de Ingeniería, J. Herrera y Reissig 565, Montevideo, Uruguay \\ 3. Center for Gravitational Physics and Geometry, Department of Physics, \\ 104 Davey Lab, The Pennsylvania State University, University Park, PA 16802
}

(July 13, 2021)

\begin{abstract}
This article reviews the status of several solutions to all the constraints of quantum gravity that have been proposed in terms of loops and extended loops. We discuss pitfalls of several of the results and in particular discuss the issues of covariance and regularization of the constraints in terms of extended loops. We also propose a formalism for "thickened out loops" which does not face the covariance problems of extended loops and may allow to regularize expressions in a consistent manner.
\end{abstract}

\section{INTRODUCTION}

The intention of this paper is to review the state of the art in February 1995 of what is perceived as one of the main achievements of the new variable canonical quantization program: the possibility to find in a generic case solutions to the Wheeler-DeWitt equation of quantum gravity. This has been one of the main motivations for the study of the new variables and led to many of its most striking results: the introduction of holonomies [1], the loop representation and its connections with knot theory [2], the connection with Chern-Simons theory and the Jones polynomial [3] and to the formulation of the extended loop representation [4]. Due to the dynamic character of research in this field many results have superseded or changed the perspective on previous ones and a review of the different problems is in order. We will try to structure this article chronologically and discuss many new aspects of old results that have been discovered with subsequent developments.

For reasons of space, this article is not self-contained. For the sake of brevity we will try to concentrate on outstanding issues, and referring the reader to appropriate references for details. The structure of this article is as follows: in section 2 we will discuss the Hamiltonian and the formulation of the theory in terms of loops. We will then discuss solutions in terms of loops based on their intersection structure and solutions based on the Jones polynomial and issues of their regularization. In section 4 we will briefly introduce the extended representation as a solution to the problem of regularizing wavefunctions and study the issue of the regularization and renormalization of the constraints. In section 5 we discuss the existence of regularized solutions and the need for counterterms in the renormalized constraints. In section 6 we will discuss the problem of the gauge covariance of the extended representation and possible solutions based on thickened out loops.

\section{THE LOOP REPRESENTATION}

Whenever one has a theory given in terms of a Lie-algebra valued connection $A_{a}^{i}$ on a three dimensional manifold, one can introduce a loop representation for it. One way of doing this is via the loop transform,

$$
\Psi(\gamma)=\int d A W_{\gamma}[A] \Psi[A]
$$

where $W_{\gamma}[A]$ ("Wilson loop") is the trace of the holonomy of the connection $A$ along the loop $\gamma$. The transform is a functional integral and we refer to the article by Ashtekar et al [5] in this volume for progress concerning its rigorous definition. Here we will use it in a heuristic way. 
It will be convenient to view wavefunctions in the loop representation $\Psi(\gamma)$ as functions on the group of loops base-pointed at a point $o, \mathcal{L}_{o}$ [6]. Because of the definition (1) these wavefunctions satisfy a series of identities. To begin with they are base-point-independent because the trace of a holonomy is. As a consequence of the properties of products of traces of matrices in a Lie-group one has the Mandelstam identities, which for the case of interest $(S U(2))$ are given by,

$$
\begin{aligned}
\Psi\left(\gamma_{1} \circ \gamma_{2}\right) & =\Psi\left(\gamma_{2} \circ \gamma_{1}\right) \\
\Psi(\gamma) & =\Psi\left(\gamma^{-1}\right) \\
\Psi\left(\gamma_{1} \circ \gamma_{2} \circ \gamma_{3}\right)+\Psi\left(\gamma_{1} \circ \gamma_{2} \circ \gamma_{3}^{-1}\right) & =\Psi\left(\gamma_{2} \circ \gamma_{1} \circ \gamma_{3}\right)+\Psi\left(\gamma_{2} \circ \gamma_{1} \circ \gamma_{3}^{-1}\right)
\end{aligned}
$$

Some comments are in order. Sometimes loop representations have been constructed based on multi-loops. In that case one considers in the transform a product of Wilson loops, one for each loop forming the multi-loop. If one proceeds that way, there are also Mandelstam identities relating wavefunctions of different multi-loops. It turns out that for the $S U(2)$ case these identities allow to express all multi-loop wavefunctions in terms of functions of single loops. We will assume such reduction has been performed and work with a single loop from now on. Notice that even with a single loop one has identities, the ones we listed above. By suitable combinations of these one can get an infinite number of identities that must be satisfied by the wavefunctions. A procedure to characterize a set of independent loops under these identities is described in the article by Rovelli and Smolin in this volume [7]. There are other properties satisfied by traces of holonomies, for instance inequalities [8.9], that reflect properties of the gauge group. It is not clear at present what are the consequences on the space of wavefunctions of there properties, so we will ignore them here. These and other problems are intimately related to the precise definition of a loop transform, as can be seen in particular examples [10] where the transform can be explicitly computed and in current efforts to define rigorously the transform.

Viewing the wavefunctions as functions of the group of loops has the advantage of immediately incorporating another symmetry of the wavefunctions, the invariance under the addition of retraced paths of vanishing area ("trees"), $\Psi(\alpha)=\Psi\left(\alpha \circ \eta_{x} \circ \eta_{x}^{-1}\right)$ where $\eta_{x}$ is an open path going from some point on the loop to the point $x$. This invariance, coupled with the Mandelstam identities, constrains the kind of functions and operators one can consider in the loop representation. Any wavefunction not complying with these identities is unacceptable, no matter what its behavior may be with respect to the operators of the theory.

An example of such a function, that had historical relevance, is the "characteristic" function of smooth loops, defined by,

$$
\Psi(\gamma)=\left\{\begin{array}{lc}
1 & \text { if } \gamma \text { is smooth and non-intersecting } \\
0 & \text { otherwise }
\end{array}\right.
$$

This function is apparently annihilated by the Hamiltonian constraint, as we will see shortly. However, such a function is not acceptable as a wavefunction of the gravitational field because it is not compatible with the Mandelstam identities. To see this, consider a smooth loop as a composition of three loops, as shown in figure $1, \gamma=\gamma_{1} \circ \gamma_{2} \circ \gamma_{3}$ and apply the Mandelstam identity (伍). It will give a combination of loops with intersections. One quickly runs into an inconsistency since $\psi(\gamma)$ is one since $\gamma$ is non-intersecting and has to be equal to something vanishing [11]. It may be possible, through the introduction of a non-trivial inner product to construct states compatible with the Mandelstam identity and related to non-intersecting loops, see 12,13. If such states are to be annihilated by the Hamiltonian constraint, it should be a self-adjoint operator with respect to the inner product introduced.

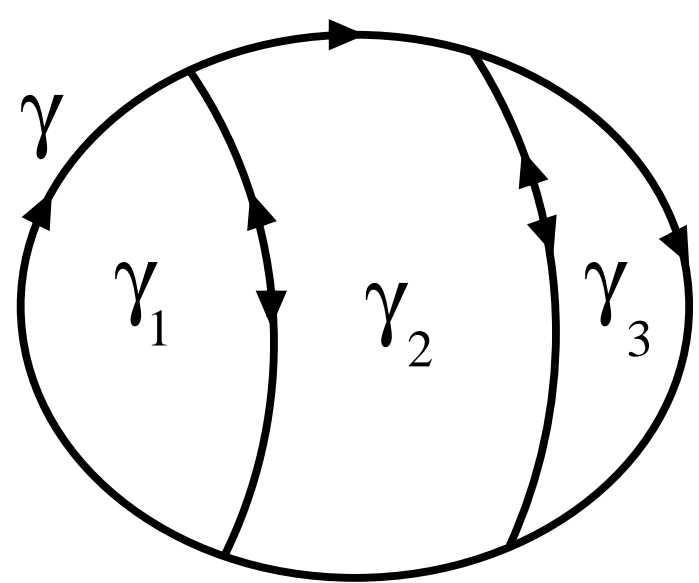

FIG. 1. The loop used in the Mandelstam identity that is not satisfied by the naive states 
Using the formal transform (11) one can obtain an expression in the loop representation for the constraints of general relativity. The expressions one gets are the following,

$$
\begin{aligned}
\mathcal{C}(\vec{N}) \Psi(\gamma) & =\int d^{3} x N^{a}(x) \oint_{\gamma} d y^{b} \delta(x-y) \Delta_{a b}\left(\gamma_{o}^{y}\right) \Psi(\gamma) \\
\mathcal{H}(\underset{\sim}{M})(x) \Psi(\gamma) & =\int d^{3} x \underset{\sim}{M} \oint_{\gamma} d y^{a} \oint_{\gamma} d z^{b} f_{\epsilon}(x-z) \delta(z-y) \Delta_{a b}\left(\gamma_{o}^{z}\right) \Psi\left(\gamma_{y}^{z} \circ \gamma_{y o}^{z}\right)
\end{aligned}
$$

These expressions have been analyzed in detail in the past 14, 15, 12 so we will not discuss them here. It has also been shown [16] that they are equivalent to the original ones introduced by Rovelli and Smolin [17]. We would like to make a few comments about them.

The first remark is that if the loop is smooth at the point in which the Hamiltonian acts, then its action is zero (we are ignoring issues related to the "acceleration terms", which are irrelevant for diffeomorphism invariant states see [12, 18]). That is the reason why the characteristic states introduced above were automatically annihilated.

The expressions we give for the constraints are written in terms of loop derivatives. The loop derivative is an operator that acts on the space of functions of base-pointed loops. Its action per se is therefore not well defined on $\Psi(\gamma)$ 's, which are not base-pointed. It is the fact that the loop derivative appears under a loop integral that makes the expression for the constraints well defined. The expressions are computed fixing a fiducial base-point in the loop and the result is independent of this choice. The first constraint has been known for some time to be the generator of infinitesimal deformations in loop space. Solutions to the first constraint are functions of loops invariant under deformations of the loops, that is, they are knot invariants. At this point another puzzle appears. Evidently the definition of the above constraints requires that the wavefunctions considered be loop differentiable. That means that they should change smoothly under the addition of an infinitesimal loop. An example of such functions would be holonomies of smooth connections. Knot invariants, however, are not in general loop differentiable. A knot invariant does not behave smoothly under deformations of the loops, its value jumps discontinuously if the deformation changes the topology of the loop. The loop derivative can do this and therefore knot invariants are in general not loop differentiable. The expressions of the constraints we introduced above will therefore require further elaboration if we want them to act on knot invariants.

The intuitive picture that therefore arises is that the diffeomorphism constraint requires us to work with wavefunctions that viewed as functions on loop space behave as "step functions"; they change values abruptly when one goes from one knot class to another. The only hope to define the action of differential operators on them in any consistent fashion is through a regularization. Notice that this is a regularization that appears in addition to the usual need to regularize the quantum operators in a field theory. It is a regularization at the level of wavefunctions.

Let us discuss these issues with some concrete examples. There exist in the literature several knot invariants for which explicit analytic expression in terms of loops can be given. These are the only invariants amenable to a concrete calculation for the action of the differential operators on them. Unfortunately none of these invariants is well defined for arbitrary loops (especially if one includes intersections). Some of these invariants are in fact not even well defined for ordinary smooth loops. An example of this is provided by the Gauss linking number of a loop with itself (the self-linking number). An analytic expression for the self-linking number is,

$$
L(\gamma)=\frac{1}{4 \pi} \oint_{\gamma} d y^{a} \oint_{\gamma} d z^{b} \epsilon_{a b c} \frac{(y-z)^{c}}{|y-z|^{3}}
$$

in extended loop notation it would be written as,

$$
L(\gamma)=-2 g_{a x b y} X^{a x b y}(\gamma)
$$

where $g_{a y b z}=-1 /(4 \pi) \epsilon_{a b c} \frac{(y-z)^{c}}{|y-z|^{3}}$ is the Green function (propagator) of a Chern-Simons theory and $X^{a x b y}$ is the second order multi-tangent associated with a loop $\gamma$ 沺. It is easy to see that as a function of a loop it satisfies the Mandelstam identities and therefore it is a candidate wavefunction for quantum gravity.

The expression (8) would be a genuine link invariant if the first and second integrals were evaluated on different loops. It would just count the linking number of both loops. In particular all information about the fiducial metric introduced to compute $|x-y|$ would be erased. When one evaluates the linking number of a loop with itself, the expression has a problem when $y=z$. In fact one can see [19] that the result of the integral is finite, but is dependent on the particular choice of fiducial metric performed to compute the Chern-Simons propagator. This is an example of what we meant by some knot invariants not being well defined even for smooth loops. If the loop has an intersection there is an added difficulty, since the resulting expression is not even finite. 
A proposed solution to this problem is to consider the invariant defined by equation (8) as an invariant of a framed loop. A framing is a procedure that given a loop assigns another loop infinitesimally separated from the original one. Another way of putting this is that it associates a preferred "ribbon" or a normal vector to a loop. The self-linking number for the framed loop is simply defined as the linking number of the two loops in the ribbon. For the self-linking number such a procedure also removes singularities in the case of intersections, since the two integrals are simply evaluated on different loops and no singularities appear. This situation is generic. Even for invariants that do not require a framing when evaluated on smooth loops (as is the case of the second coefficient of the Conway polynomial defined in [20]) one requires some procedure to remove singularities generated by intersections. We call all these procedures "framings". (More discussion on framings can be found in references [21,20]).

How would one proceed to define the action of the Hamiltonian constraint introduced above on invariants as the ones we have been discussing? Two possibilities immediately come to mind, associated with taking in different orders the two limits involved in defining the framing and the loop derivative: 1) To act with the loop derivative on the invariant evaluated for a framed loop, for a finite separation of the framing; 2) To act with the loop derivative on a framed loop in such a way that the action of the derivative could change the relative knotting of the two loops in the framing.

In the first case the action of the loop derivative is trivial. It acts on each loop individually and does not modify their relative linking. If one were to pursue this seriously any invariant would be annihilated by all the constraints. This does not seem a sensible choice, since there is no reason why any knot invariant should be annihilated by the Hamiltonian constraint. The other possibility, in which the loop derivative can change the relative topology of both loops in the framing has the disadvantage of being ill defined. The problem is that one could imagine situations in which the result of the action of the derivative would be dependent on the particular details of the loop appended in order to compute the loop derivative. It is convenient to recall that in the definition of the loop derivative, the shape of the loop did not matter as long as it spanned a certain area. This is not the case for the action of the Hamiltonian we obtain.

Is this just a problem of these particular wavefunctions or is it generic? What happens if one considers a function of loops that is well defined without introduction of a framing? As we mentioned above, the loop derivative is simply not defined. Is this therefore a problem of the particular way of writing the constraint in terms of the loop derivatives? Could one rearrange limits in such a way that the Hamiltonian has an action that is well defined on knots? A recent paper 22] has explored these and other issues. The conclusion of that particular analysis is that one can (ignoring certain subtleties [18]) find an operator that reduces in the connection representation to the Hamiltonian constraint and that has an action on knots. The trouble is that the action inherits one of the difficulties mentioned above. It corresponds to the addition of a small loop and the result therefore depends on how one adds that loop. One started with an operator that did not know about this small loop and ends up with a result that is dependent on it. It may be the case that this is inevitable and is just a reflection of the infinitely many choices one has when defining a regularization of an operator in a quantum field theory. At the moment the status of this problem is unclear.

Here we will explore yet another option. We will consider knot invariants given by expressions similar to the selflinking number we considered above. We will study the action of the Hamiltonian defined in the following way. Given an invariant generically written as " $g \cdot X(\gamma)$ " where $g$ is a set of propagators and $X(\gamma)$ is a multi-tangent of arbitrary order, we will act with the Hamiltonian on $X(\gamma)$ and compute the result. Notice that the loop derivative of the $X(\gamma)$ is well defined, so this seems to bypass the problem of differentiating a knot invariant. This is not the case since the contraction with the $g$ 's and the resulting action of the Hamiltonian are singular. The calculations we will perform are therefore purely formal. As a result we will find that several quite nontrivial knot invariants are annihilated formally by the Hamiltonian constraint. The extended loop representation which we discuss in the next section will provide an arena in which completely equivalent results appear in a fully regularized context.

There is a result in the connection representation that is the key to all the solutions we will discuss. As was first observed by Kodama [23], if one considers a wavefunction given by the exponential of the Chern-Simons form of the Ashtekar connection,

$$
\Psi_{\Lambda}[A]=\exp \left(-\frac{6}{\Lambda} S_{C S}\right) \equiv \exp \left(-\frac{6}{\Lambda} \int \tilde{\eta}^{a b c} \operatorname{Tr}\left[A_{a} \partial_{b} A_{c}+\frac{2}{3} A_{a} A_{b} A_{c}\right]\right)
$$

it is a solution of all the constraints of quantum gravity with a cosmological constant $\Lambda$ (there is a factor ordering issue with respect to the diffeomorphism constraint that was solved in [3]). If one considers the transform of this state, one obtains an integral

$$
\Psi[\gamma]=\int d A \quad W_{\gamma}[A] e^{-\frac{6}{\Lambda} S_{C S}},
$$

that formally looks the same as the expectation value of a Wilson loop in a Chern-Simons theory. This expression has been studied by many authors [21,24,20] and the result is that it is a knot invariant that is known as the Kauffman 
bracket knot polynomial. Since the integral (11) can be computed using perturbation theory (since Chern-Simons theory is renormalizable) one actually can obtain concrete expressions for the different coefficients of the Kauffman bracket.

If one believes these results one is forced therefore to conclude that the Kauffman bracket should be annihilated by the Hamiltonian constraint of quantum gravity with cosmological constant in the loop representation. This is a calculation that can be checked, at least at the formal level discussed above. The main result is that it is in fact a solution and as a surprising consequence of the calculation one finds that certain portion of the coefficients of the Kauffman bracket, which coincides precisely with a coefficient of the Jones polynomial, are annihilated by Hamiltonian constraint with $\Lambda=0$. The Kauffman bracket is known to be related to the Jones polynomial (in the arbitrary variable q) through the expression,

$$
\operatorname{Kauffman}(\gamma)_{q}=q^{\frac{3}{4} L(\gamma)} \operatorname{Jones}(\gamma)_{q},
$$

which highlights the fact that the framing dependence of the Kauffman bracket is concentrated in the prefactor involving the self linking number, since the Jones polynomial is framing independent.

Unfortunately, an initial conjecture that suggested that all the coefficients of the Jones polynomial were solutions turned out not to be true. Detailed calculations show that the third coefficient of the Jones polynomial is not annihilated by the vacuum Hamiltonian constraint. This is in spite of the fact that the Kauffman bracket is annihilated up to that order. In pursuing this analysis it was also noted [15] that at the root of the result is the fact that the prefactor is formally a solution of the Hamiltonian constraint with cosmological constant. It can be checked that all these states satisfy the Mandelstam identities and solve the constraints without any assumption about the loops (they can have an arbitrary number of intersections).

In spite of the fact of the nontrivial nature of all these results, which arise due to elaborate cancelations of terms in the action of the Hamiltonian, we remind the reader that the results are all formal in the sense discussed above. This non-triviality of the results is the main motivation to seek a framework that would allow to make sense of them in a fully regularized context.

\section{THE EXTENDED LOOP REPRESENTATION}

The extended loop representation is obtained by replacing the multi-tangents (also called "form factors") that appear in the definition of the holonomy by arbitrary multi-vector densities. What is the point of doing this? Consider the expression we introduced in the previous section for the self linking number. If instead of being given by a double loop integral it had been given by a double spatial integral along smooth vector densities $X^{a}(y)$,

$$
L(X)=\frac{1}{4 \pi} \int d^{3} y \int d^{3} z X^{a}(y) X^{b}(z) \epsilon_{a b c} \frac{(y-z)^{c}}{|y-z|^{3}}
$$

the integral would have been well defined. The above integral yields the usual expression if one replaces the smooth vector densities by the distributional tangents to the loops $X^{a}(y)(\gamma)=X^{a y}(\gamma) \equiv \oint_{\gamma} d z^{a} \delta(z-y)$. The problem we are confronted with is analogous to the usual difficulty in electrostatics: if one computes the energy of a set of distributional charges a divergence appears if the charges touch. If the distributions are smooth no problem arises in spite of the fact that the propagator is distributional.

The rationale for extended loops is therefore to replace the ill definitions that arise in terms of loops due to their distributional nature by replacing the information needed about the loops tangents by smooth fields. Doing this in the non-Abelian case requires some detailed work that we briefly discuss. More complete treatments can be found in [4, 25].

The starting point is the expression for the "extended holonomy",

$$
U(\mathbf{X})=\mathbf{X} \cdot \mathbf{A} \equiv \sum_{n=0}^{\infty} X_{\sim}^{\mu_{n}} A_{\mu_{n}}
$$

where $X_{\sim}^{\mu_{n}}$ is a shorthand for $X^{a_{1} x_{1}, \ldots, a_{n} x_{n}}$ and $A_{\sim_{\sim}}$ for $A_{a_{1}}\left(x_{1}\right) \cdots A_{a_{n}}\left(x_{n}\right)$ and $X$ is a set of multivector densities. If one would like to recover the usual holonomy of a loop one needs to replace the $X$ 's by appropriate multi-tangents to the loop, details are given in $[$.

Obviously such an expression does not in general converge. It does not seem reasonable to discuss convergence criteria for it, since in general it is not even gauge covariant. If one requires (formally) that the extended holonomy have a gauge invariant trace one needs to require certain conditions on the multi-tensors, 


$$
\begin{aligned}
& \frac{\partial}{\partial x_{i}^{a_{i}}} X^{a_{1} x_{1} \ldots a_{i} x_{i} \ldots a_{n} x_{n}}= \\
& \quad\left(\delta\left(x_{i}-x_{i-1}\right)-\delta\left(x_{i}-x_{i+1}\right)\right) X^{a_{1} x_{1} \ldots a_{i-1} x_{i-1} a_{i+1} x_{i+1} \ldots a_{n} x_{n}} .
\end{aligned}
$$

which are usually referred to as "the differential constraint". In this expression, points $x_{0}$ and $x_{n+1}$ are to be understood as the base-point of the loop. The multi-tangents of a usual loop satisfy this equation.

Now that we know how to obtain gauge invariants from extended loops, can we answer in a meaningful way the question of the convergence of the extended holonomy? The answer is again negative. Although the trace of the whole series is gauge invariant, each individual term is not. The usual way to determine if a series converges is by comparing successive terms in the series. Such criteria therefore cannot be translated into a gauge invariant statement. We will therefore simply have to assume that the extended loops we are dealing with are those for which the extended holonomy converges for the portion of the space of connections modulo gauge transformations of physical interest.

Even assuming this, in which sense does the differential constraint ensure that the trace of the extended holonomy is gauge invariant? If one performs an infinitesimal gauge transformation of the extended holonomy one gets an infinite series. The differential constraint ensures that terms in that series cancel in pairs. Of course, this only implies gauge invariance given certain properties of convergence of the series. The precise statement is that the term [26],

$$
\sum_{k=1}^{N} A_{\mu_{1}} \ldots A_{\mu_{k-1}}[A, \Lambda]_{\mu_{k}} A_{\mu_{k+1}} \ldots A_{\mu_{n}} X^{\mu_{1} \ldots \mu_{n}}
$$

should go to zero as $N \rightarrow \infty$. $\Lambda$ is the parameter of the gauge transformation and is therefore an arbitrary function. Notice that the vanishing of (16) is not guaranteed by the convergence of the holonomy alone.

These problems of convergence were illustrated in a recent paper by by Schilling [26] where explicit examples of extended loops that satisfied the differential constraint and that failed to give extended holonomies with invariant trace were constructed. Does this mean that the formalism is doomed? We believe the answer is no. We will show in section 5 that one can actually find a large subset of extended loops for which the convergence problems do not arise and for which the following framework would be applicable.

Another comment about the differential constraint is that due to its structure it mandates that the multi-tangents should be distributional objects. Does this defeat the whole purpose of extended loops? After all, we introduced them in order to smoothen out the distributional behavior of ordinary loops. We will see that the following set of ideas allows us to make use of these objects, in spite of the fact that they are distributional.

The $X$ 's that satisfy (15) form a group with composition law,

$$
\left(\mathbf{X}_{1} \times \mathbf{X}_{1}\right)^{\mu_{n}} \equiv \sum_{k=1}^{n} X_{1}^{\mu_{1}, \ldots, \mu_{k}} X_{2}^{\mu_{k+1}, \ldots, \mu_{n}}
$$

where $\mu_{i}$ stands for a paired vector index and spatial point $a_{i}, x_{i}$. This group is called the extended group of loops. The ordinary group of loops arises in the limit when one replaces $X$ 's by the multi-tangents to a loop. Associated with the extended group is an infinite dimensional Lie algebra. Due to this one can write a generic element of the extended group of loops as,

$$
\mathbf{X}=\exp (\sigma \cdot \mathbf{Y})
$$

where the exponential is determined by the product introduced above, the $\sigma$ 's are a basis for the algebra given explicitly in [4] and $Y$ is an arbitrary multitensor. We omit all details. The point is that because the $Y$ 's are arbitrary, one in particular can choose them to be smooth. As a consequence of this we see that all the distributional structure of the $X$ 's has to arise from the $\sigma$ 's. Since these are known explicitly, this structure is well under control. We will see that this explicit knowledge of the singularity structure is a powerful aid in regularizing the wavefunctions and the action of the constraints on them.

In fact if one considers the wavefunctions given by the knot invariants explicitly introduced in the previous section from Chern-Simons theory, the ones that looked like " $\mathrm{g} \cdot \mathbf{X}$ ", it can be explicitly checked [25] that the particular form of the $\mathbf{g}^{\prime} s$ that comes from Chern-Simons theory is such that if one introduces the $X$ 's stemming from smooth $Y$ 's introduced above, all the divergent structure is annihilated and one is left with a well defined smooth expression purely given in terms of the $Y$ 's. This is due to the particular form and symmetries of the propagators that appear for these wavefunctions contracted with the $X$ 's. Therefore we see that the extended loops have delivered their promise: due to their less singular nature than loops they manage to provide expressions for the gauge invariants that are well defined. 
In order to write expressions for the constraints in the extended representation it is worthwhile to notice that due to the Mandelstam identities wavefunctions only depend on the extended loops through the combination,

$$
R^{\mu_{1} \ldots \mu_{n}}=\frac{1}{2}\left[X^{\mu_{1} \ldots \mu_{n}}+(-1)^{n} X^{\mu_{n} \ldots \mu_{1}}\right]
$$

Acting on functions of $R$ the constraints can be written as linear functional operators (for details see 25]),

$$
\begin{aligned}
\mathcal{C}(\vec{N}) \Psi(\mathbf{R}) & =\int d^{3} x N^{a}(x)\left(\mathcal{F}_{a b}(x) \times \mathbf{R}^{(b x)}\right) \stackrel{\mu}{\sim} \frac{\delta}{\delta \mathbf{R} \sim} \Psi(\mathbf{R}) \\
\mathcal{H}(\underset{\sim}{\sim}) & =2 \int d^{3} x \underset{\sim}{\sim}(x)\left(\mathcal{F}_{a b}(x) \times \mathbf{R}^{(a x, b x)}\right) \stackrel{\mu}{\sim} \frac{\delta}{\delta \mathbf{R}^{\mu}} \Psi(\mathbf{R})
\end{aligned}
$$

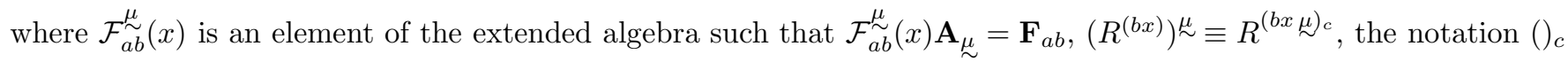
meaning cyclic permutation of indices. The element $\mathbf{R}^{(a x, b x)}$ is given by

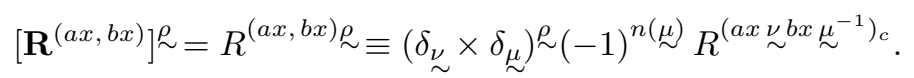

It is remarkable that the operators have such a similar action, though the notation helps to conceal important differences. They are basically concentrated in the special elements with "marked" points $x$ that accompany the $\mathcal{F}_{a b}$ in the expression of the constraints. The element $\mathbf{R}^{(a x, b x)}$ is evidently divergent since it has a spatial dependence repeated and we know through the differential constraint that the object is distributional. That is the reason why the Hamiltonian still requires a regularization in spite of being a linear operator. A point-splitting regularization is simply achieved by separating the two $x$ 's in the $R^{(a x, b x)}$.

As we discussed above, the wavefunctions we introduced in the previous section are simply generalized to the extended representation by formally substituting the dependence on the multi-tangents to a loop by the dependence on an arbitrary multitensor stemming from a smooth Y. Let us exhibit this explicitly for the second coefficient of the Alexander-Conway polynomial. Its expression in terms of multi-tangents fields is [20],

$$
a_{2}[\gamma]=h_{\mu_{1} \mu_{2} \mu_{3}} X^{\mu_{1} \mu_{2} \mu_{3}}(\gamma)+g_{\mu_{1} \mu_{3}} g_{\mu_{2} \mu_{4}} X^{\mu_{1} \mu_{2} \mu_{3} \mu_{4}}(\gamma)
$$

where

$$
h_{\mu_{1} \mu_{2} \mu_{3}}=\int d^{3} z \epsilon^{b_{1} b_{2} b_{3}} g_{\mu_{1} b_{1} z} g_{\mu_{2} b_{2} z} g_{\mu_{3} b_{3} z}
$$

with

$$
\epsilon^{c_{1} z_{1} c_{2} z_{2} c_{3} z_{3}}=\epsilon^{c_{1} c_{2} c_{3}} \int d^{3} t \delta\left(z_{1}-t\right) \delta\left(z_{2}-t\right) \delta\left(z_{3}-t\right)
$$

The generalization of this knot invariant to extended loops is straightforward

$$
a_{2}[\gamma]=a_{2}[\mathbf{X}(\gamma)] \rightarrow a_{2}(\mathbf{X})=a_{2}(\mathbf{R})
$$

We now analyze the application of the Hamiltonian constraint on this state in the extended representation. With the expression introduced above we have,

$$
\begin{array}{r}
\mathcal{H}(x) a_{2}(\mathbf{R})=2 h_{\mu_{1} \mu_{2} \mu_{3}}\left[\mathcal{F}_{a b} \mu_{1}(x) R^{(a x, b x) \mu_{2} \mu_{3}}+\mathcal{F}_{a b} \mu_{1} \mu_{2}(x) R^{(a x, b x) \mu_{3}}\right] \\
+2 g_{\mu_{1} \mu_{3}} g_{\mu_{2} \mu_{4}}\left[\mathcal{F}_{a b} \mu_{1}(x) R^{(a x, b x) \mu_{2} \mu_{3} \mu_{4}}+\mathcal{F}_{a b} \mu_{1} \mu_{2}(x) R^{(a x, b x) \mu_{3} \mu_{4}}\right]
\end{array}
$$

We can compute the action of $\mathcal{F}_{a b}$ on the propagators $g$ by integration by parts and the use of their explicit expressions. The results are,

$$
\begin{aligned}
& \mathcal{F}_{a b}{ }^{\mu_{1}}(x) g_{\mu_{1} \mu_{3}}=-\epsilon_{a b a_{3}} \delta\left(x-x_{3}\right)-\partial_{a_{3}} g_{a x} b x_{3} \\
& \mathcal{F}_{a b} \mu_{1} \mu_{2}(x) g_{\mu_{1} \mu_{3}} g_{\mu_{2} \mu_{4}}=g_{\mu_{3}[a x} g_{b x] \mu_{4}} \\
& \begin{aligned}
\mathcal{F}_{a b}{ }^{\mu_{1}}(x) h_{\mu_{1} \mu_{2} \mu_{3}}= & -g_{\mu_{2}[a x} g_{b x] \mu_{3}}+\left(g_{a x b x_{2}}-g_{a x b x_{3}}\right) g_{\mu_{2} \mu_{3}} \\
& +\frac{1}{2} g_{a x b z} \epsilon^{d e f}\left[g_{\mu_{3} d z} \partial_{a_{2}} g_{e x_{2} f z}-g_{\mu_{2} d z} \partial_{a_{3}} g_{e x_{3} f z}\right] \\
\mathcal{F}_{a b} \mu_{1} \mu_{2}(x) h_{\mu_{1} \mu_{2} \mu_{3}}= & 2 h_{a x b x \mu_{3}}
\end{aligned}
\end{aligned}
$$


In the last term of equation (30) an implicit integral in $z$ is assumed. The derivatives that appear in the above expressions can be applied over the R's integrating by parts, and using the differential constraint we generate from them terms of lower rank in $R$. For example from (28) we have

$$
g_{\mu_{2} \mu_{4}} \partial_{a_{3}} g_{a x b x_{3}} R^{(a x, b x) \mu_{2} \mu_{3} \mu_{4}}=g_{\mu_{2} \mu_{4}}\left(g_{a x b x_{2}}-g_{a x b x_{4}}\right) R^{(a x, b x) \mu_{2} \mu_{4}}
$$

Performing these calculations, the following partial results are obtained for each of the four expressions quoted above:

$$
\begin{aligned}
& \text { 1) }-\epsilon_{a b c} g_{\mu_{1} \mu_{2}} R^{(a x, b x) \mu_{1} c x \mu_{2}}-\left(g_{a x b x_{1}}-g_{a x b x_{2}}\right) g_{\mu_{1} \mu_{2}} R^{(a x, b x) \mu_{1} \mu_{2}} \\
& \text { 2) } g_{\mu_{1}[a x} g_{b x] \mu_{2}} R^{(a x, b x) \mu_{1} \mu_{2}} \\
& \text { 3) }-g_{\mu_{1}[a x} g_{b x] \mu_{2}} R^{(a x, b x) \mu_{1} \mu_{2}}+\left(g_{a x b x_{1}}-g_{a x b x_{2}}\right) g_{\mu_{1} \mu_{2}} R^{(a x, b x) \mu_{1} \mu_{2}} \\
& -\epsilon^{\text {def }} g_{a x b z} g_{\mu_{1} d z} g_{e x f z} R^{(a x, b x) \mu_{1}} \\
& \text { 4) } 2 h_{a x b x \mu_{1}} R^{(a x, b x) \mu_{1}}
\end{aligned}
$$

Some contributions cancel each other. Integrating by parts the last term and using the differential constraint we finally obtain

$$
\begin{aligned}
\mathcal{H}(x) a_{2}(\mathbf{R})= & -2 \epsilon_{a b c} g_{\mu_{1} \mu_{2}} R^{(a x, b x) \mu_{1} c x \mu_{2}} \\
& +2\left[2 h_{a x} b x \mu_{1}-\epsilon^{d e f} g_{a x b z} g_{\mu_{1} d z} g_{e x f z}\right] R^{(a x, b x) \mu_{1}}
\end{aligned}
$$

In the above expression, it can be easily checked that the square bracket vanishes identically. Expanding $R^{(a x, b x) \mu_{1} c x \mu_{2}}$ we get

$$
R^{(a x, b x) \mu_{1} c x \mu_{2}}=-2 R^{\left(a x b x \mu_{1} c x \mu_{2}\right)_{c}}+R^{\left(c x a x \mu_{1} b x \mu_{2}\right)_{c}}+R^{\left(b x c x \mu_{1} a x \mu_{2}\right)_{c}}
$$

and we therefore see that the contribution of the first term vanishes due to symmetry considerations. We conclude that

$$
\mathcal{H}(x) a_{2}(\mathbf{R})=0
$$

We see that the explicit computation of this formal result in the extended representation involves only a few simple steps. It shows what a powerful computational tool the use of extended loops provides us with, just by comparing with the difficult nature of the same computation done in terms of loops [27]. The extended loop analogue of the second coefficient of the Alexander-Conway polynomial is therefore annihilated formally by all the constraints of quantum gravity in the extended loop representation. We will now discuss the same derivation in a fully regularized context.

\section{REGULARIZED SOLUTIONS BASED ON EXTENDED LOOPS}

As we discussed above, one is forced to regularized the Hamiltonian constraint to avoid divergences. In order to do that one has to point-split the expression where repeated spatial points appear in a multitensor. The regularized expression for the constraint therefore is,

$$
\mathcal{H}^{\epsilon}(x) \psi(\mathbf{R})=2 \int d^{3} w \int d^{3} u \int d^{3} v f_{\epsilon}(w, x) f_{\epsilon}(u, x) f_{\epsilon}(v, x) \psi\left(\mathcal{F}_{a b}(w) \times \mathbf{R}^{(a u, b v)}\right) .
$$

If one now applies this constraint to the state we considered in the previous section, one can check that all the computations followed up (but excluding ) expression (33) are well defined and all cancellations we discussed take place rigorously. The main difference arises when we integrated by parts the last contribution to (28) and applied the differential constraint. Because now one is dealing with a regularized object, $\mathbf{R}^{(a x, b y)}$ does not satisfy the differential constraint basepointed at $x$ any longer. The resulting expression for the action of the constraint is,

$$
\begin{array}{r}
\mathcal{H}^{\epsilon}(x) a_{2}(\mathbf{R})=\int d^{3} w \int d^{3} u \int d^{3} v f_{\epsilon}(w, x) f_{\epsilon}(u, x) f_{\epsilon}(v, x) \\
\left\{-\epsilon_{a b c} g_{\mu_{1} \mu_{2}} R^{(a u, b v) \mu_{1} c w \mu_{2}}+\right. \\
{\left[2 h_{a w b w \mu_{1}}-\epsilon^{d e f} g_{a w b z} g_{\mu_{1} d z} g_{e u f z}\right] R^{(a u, b v) \mu_{1}}+} \\
\left.\quad\left(g_{a w b u}-g_{a w b v}\right) g_{\mu_{1} \mu_{2}} R^{\left(a u \mu_{1} b v \mu_{2}\right)_{c}}\right\} .
\end{array}
$$


The first two terms cancel for the same reason a before, but one is left with the last term (which we call "anomalous term"). If one studies in detail the nature of this term when one removes the regulator one finds a contribution of order $1 / \epsilon$,

$$
\left.\frac{2}{\sqrt{2 \pi} \epsilon} \epsilon_{a b c} g_{\mu_{1} \mu_{2}} \partial^{c y} R^{\left(a x \mu_{1} b y \mu_{2}\right)_{c}}\right|_{y=x}
$$

if one assumes that the regulator is Gaussian $f_{\epsilon}(z)=(\sqrt{\pi} \epsilon)^{-3} \exp \left(-z^{2} \epsilon^{-2}\right)$.

If one renormalizes the Hamiltonian by $\epsilon$ we see that the state is not annihilated by the constraint. Similar results can be derived for all other states introduced in section III, the Kauffman bracket and the exponential of the self-linking number.

Is this the end of the story? Are the states introduced just artifacts of the formal calculations that do not survive the introduction of a regularization? We would like to claim that the answer is no. When one works with regularized expressions one should open up the possibility that corrections may arise such that in the limit in which regulators are removed the corrections are zero assuming certain regularity properties of the wavefunctions. Consider for instance the action of the operator,

$$
C(u, v)=R^{\left(a u \underset{\sim}{\mu} b v \nu_{\sim}\right)_{c}}\left(\frac{\delta}{\delta R^{(a w \mu b u)} \underset{\sim}{\nu}}-\frac{\delta}{\delta R^{(a w \mu \nu b v) \nu}}\right)
$$

on a function of extended loops $\Psi(\mathbf{R})=\mathbf{D} \cdot \mathbf{R}$ with $\mathbf{D}$ smooth (an example of these kinds of functions would be the holonomy of a smooth connection). Evidently the action vanishes due to symmetry arguments in the limit in which $u \rightarrow v$. However if one considers distributional $\mathbf{D}^{\prime} s$ (as we have done in the explicit expressions for the knot invariants we propose as possible solutions ) the action is not obviously vanishing anymore since in the limit $u \rightarrow v$ one gets a subtraction of two infinities. Expressions like this one generate "anomalous"-like terms,

$$
C^{\epsilon}\left(g_{\mu_{1} \mu_{2}} R^{\mu_{1} \mu_{2}}\right)=2\left(g_{a w b u}-g_{a w b v}\right) R^{(a u b v)_{c}}
$$

Could it be that adding expressions like the above one to the Hamiltonian one can cancel the anomalous terms? The answer is in the affirmative. The precise counter-term is given by the difference of two terms, $\mathcal{C}_{2}-\mathcal{C}_{1}$,

$$
\begin{aligned}
& \mathcal{C}_{1}=R^{(a u \underset{\sim}{\mu} b v \underset{\sim}{\nu})_{c}}\left(\frac{\delta}{\delta R^{a w} \underset{\sim}{\mu b u \nu} \sim}-\frac{\delta}{\delta R^{a w \mu} \underset{\sim}{\sim} b v \underset{\sim}{\sim}}\right) \\
& \mathcal{C}_{2}=\left(R^{(a u b v) \underset{\sim}{\sim}}+\frac{1}{2} R^{[a u b v] \underset{\sim}{\alpha}}\right)\left(\frac{\delta}{\delta R^{(a w b u)_{c} \alpha} \sim}-\frac{\delta}{\delta R^{(a w b v)_{c} \alpha}}\right) .
\end{aligned}
$$

where the expression $R^{[a u b v] \underset{\sim}{\sim}}$ is the given by expression $(22)$ without the $(-1)^{n(\mu)}$ factor and without the "rerouting" action (the index $\mu^{-1}$ is replaced by $\mu$. Remarkably, these expressions also have a simple form in the connection representation,

$$
\begin{aligned}
& \mathcal{C}_{1}:=\left(A_{a w}^{i} A_{b u}^{k}-A_{a w}^{i} A_{b v}^{k}\right) \frac{\delta}{\delta A_{a u}^{i}} \frac{\delta}{\delta A_{b v}^{k}} \\
& \mathcal{C}_{2}:=\left(A_{a w}^{i} A_{b u}^{i}-A_{a w}^{i} A_{b v}^{i}\right) \frac{\delta}{\delta A_{a u}^{k}} \frac{\delta}{\delta A_{b v}^{k}}
\end{aligned}
$$

With this single counter-term all the anomalous contributions to the action of the Hamiltonian constraint on the $a_{2}$, the Kauffman bracket and the exponential of the self-linking number cancel. The fact that a single counterterm is responsible for all the cancellations is remarkable and shows that the construction is not just a gimmick to fix the anomaly problem, but might well be a genuine counter-term arising from quantum gravity. The fact that the counter-term has a simple and precise expression in the connection representation raises the hope that a better intuitive explanation of it could be gained by viewing it in this context. At present this issue is not settled: could it be that $\mathcal{C}_{2}-\mathcal{C}_{1}$ is what one needs to add to the Hamiltonian in the connection representation in order to annihilate the exponential of the Chern-Simons form when distributional connections are allowed? Could it reflect the fact that in that case a nontrivial contribution to the measure arises? These issues are currently being studied.

\section{COVARIANCE OF THE EXTENDED LOOP APPROACH}

Due to the results of the previous section, it appears that the extended representation is a fruitful avenue to regularize quantum gravity. The fact that quite nontrivial states are found to be regularized solutions to the constraints is a 
strong motivation to continue the study of extended loops. The only obstacle to the use of extended loops as an arena to quantize gravity seems to stem from the objection that we mentioned at the beginning concerning convergence issues for the extended holonomy.

The question of what is a suitable domain in the space of extended loops to consider for the quantization of gravity so that the covariance of the formulation under gauge transformations is preserved is a hard question involving functional spaces. It is by no means settled at present. What we would like to do in this section is to study several aspects of these covariance issues. We will conclude it with a definition of a set of objects based on extended loops for which no covariance problem arises, to which we can apply the formalism of the previous sections and which include ordinary loops as a limiting case.

\section{A. The power series holonomy and convergence issues}

In the previous sections we reviewed the construction of a Lie group structure that includes loops as a particular case, the extended group of loops. The main motivation to consider the study of loops has been the construction of holonomies along the loops given a connection. Holonomies can be viewed as a Lie group-valued representation of the group of loops, the Lie group being given by the gauge group. For the extended group of loops one can consider similar representations, typically from the extended group to the enveloping group of the gauge group (the most general group associated with the dimension of the representation chosen for the Lie group; for instance, for SU(2) it would be $\mathrm{GL}(2, \mathrm{C})$ ). One example of such map, which has been considered up to present in the literature as the "extended holonomy" is simply given by contracting an element of the group of loops,

$$
U(\mathbf{X})=\mathbf{X} \cdot \mathbf{A}
$$

which formally satisfies $U\left(\mathbf{X}_{1} \times \mathbf{X}_{2}\right)=U\left(\mathbf{X}_{1}\right) U\left(\mathbf{X}_{2}\right)$.

To distinguish this expression from other possible candidates for a holonomy we will in this paper call it "power series holonomy".

The above expression implies an infinite summation and its convergence has to be studied in detail. It should be remarked that this is the case already for the usual holonomy. The usual holonomy of a connection along a loop is given by,

$$
U_{A}(\gamma)=\lim _{n \rightarrow \infty} \prod_{i=1}^{n}\left(1+\delta x_{i}^{a} \mathbf{A}_{a}\right)
$$

and the limit appearing in the product is only well-defined for particular connections, which we will call "smooth". More precisely, we will limit ourselves to connections such that the above expression can be re-expressed as in equation (43) with $X=X(\gamma)$, the multi-tangents of a loop for all piecewise-smooth loops $\gamma$.

As we noticed, dealing with power-series extended holonomies requires some detailed understanding of the convergence properties of the infinite summations involved. For example, a condition for an extended holonomy to furnish a correct representation of the extended group of loops, the holonomy of the product of two extended loops should equal the product of the holonomies of the individual extended loops. This in particular implies that,

$$
\begin{aligned}
& U\left(\mathbf{X}_{1} \times \mathbf{X}_{2}\right)=\lim _{n \rightarrow \infty} \sum_{k=0}^{n} A_{\mu_{1}, \ldots, \mu_{k}}\left(X_{1} \times X_{2}\right)^{\mu_{1}, \ldots, \mu_{k}} \\
& =\lim _{n \rightarrow \infty} \sum_{k=0}^{n} \sum_{i=0}^{k} A_{\mu_{1}, \ldots, \mu_{k}} X_{1}^{\mu_{1}, \ldots, \mu_{i}} X_{2}^{\mu_{i+1}, \ldots, \mu_{k}} \\
& =\lim _{\substack{n \rightarrow \infty \\
m \rightarrow \infty}} \sum_{k=1}^{n} \sum_{i=1}^{m} A_{\mu_{1}, \ldots, \mu_{k}} X_{1}^{\mu_{1}, \ldots, \mu_{k}} A_{\nu_{1}, \ldots, \nu_{i}} X_{2}^{\nu_{1}, \ldots, \nu_{i}} \\
& =U\left(\mathbf{X}_{1}\right) U\left(\mathbf{X}_{2}\right) .
\end{aligned}
$$

The equality between (46) and $(47)$ is not automatic, one needs certain assumptions about the convergence of the series involved. For ordinary loops the identity holds, since as we mentioned we are considering connections such that the usual holonomies furnish a representation of the group of loops.

The notion of power-series holonomy we have just defined has the undesirable property of not being gauge invariant for all elements of the extended group of loops that satisfy the differential constraint. This was discussed in detail 
in reference [26]. Basically it was observed that if one considered an element of the extended group obtained as the non-rational power of a loop the power-series holonomy was not gauge invariant. These explicit examples showed clearly how dangerous it is to take for granted the convergence of many of the expressions one considers. Although formally it is true that if an extended loop satisfied the differential constraint, the resulting power-series holonomy as defined above is gauge invariant, in many practical examples the formal proof fails due to convergence difficulties.

There are two different aspects we would like to highlight concerning these convergence issues. One of them is which are the requirements for a holonomy of the type $\mathbf{X} \cdot \mathbf{A}$ to be well defined and under which circumstances other definitions of holonomy may be needed in order to ensure gauge invariance.

\section{B. Examples of extended loops for which the power-series holonomy is well defined}

When the examples of non-covariant holonomies were first discovered [26] a concern was raised that maybe the only elements of the extended group of loops for which the holonomy was well defined were the ordinary loops. In this subsection we will prove two main results:

a) that there exist many elements of the extended group that are more general than ordinary loops for which the power-series holonomy is well defined,

b) that some of these elements form a Lie-group structure. These objects will not include ordinary loops as a particular case, if one insists that their power-series holonomy be gauge invariant. We will discuss in the following subsection how to define other notions of holonomy that would allow us to recover ordinary loops as a particular case. We will see that these new notions of holonomy can be viewed as "analytic continuations" of the power-series holonomy outside of its domain of convergence.

The most trivial example of an element of the group of loops for which the power-series holonomy is well-defined is furnish by the algebra of elements first considered by Giles [28] which is simply obtained by linear superpositions of the multi-tangents of ordinary loops. Given a finite family $\Gamma$ of $N$ loops $\gamma_{i}$, one defines a linear combination

$$
X(\Gamma, \vec{\alpha})=\sum_{i}^{N} \alpha_{i} \mathbf{X}\left(\gamma_{i}\right),
$$

and these summations immediately satisfy the differential constraint since each of the individual $\mathbf{X}\left(\gamma_{i}\right)$ 's does and the differential constraint is a linear equation. This leads to the power-series holonomy,

$$
U(\Gamma, \vec{\alpha})=\sum_{i}^{N} \alpha_{i} U\left(\gamma_{i}\right) .
$$

Each of the individual $U$ 's in the above summation is an element of the gauge group, since they are simply holonomies along ordinary loops. However, the resulting sum will not be an element of the gauge group, but of the algebra of $n \times n$ complex matrices if the representation of the gauge group is in terms of $n \times n$ matrices. This is not surprising, since one knows that for the power-series holonomy to belong to the gauge group, the element of the extended group should satisfy an additional restriction [1], called "the algebraic constraint", and this is a nonlinear equation and therefore not satisfied in general by the above linear superpositions. Although one could arrange for some of the examples we will discuss to satisfy the algebraic constraint, we will for simplicity ignore this issue. The reason for this is that for the purpose of constructing extended representations one is mainly interested in producing holonomies whose trace is gauge invariant and for that it suffices to satisfy the differential constraint only.

The above set of elements does not in general form a group. The reason for this is that the elements generated are not in general invertible. One can gain invertibility by imposing,

$$
\sum_{i=1}^{N} \alpha_{i}=1
$$

The inverse of an element of the extended group of loops is given by,

$$
\mathbf{X}^{-1}=X^{-1} \mathbf{I}+\sum_{i=1}^{\infty}(-1)^{i} X^{-i-1}(\mathbf{X}-X \mathbf{I})^{i}
$$

and the condition (50), which is equivalent to $X=1$ assures that the above expression is well defined. Notice that the above expression involves only a finite sum for each component of the element $\mathbf{X}$. We also see that the power-series 
holonomy of such an element involves an infinite expansion since one adds up all components of the extended element. If one explicitly computes it, one gets,

$$
U\left(\mathbf{X}^{-1}(\Gamma, \vec{\alpha})\right)=I+\sum_{i=1}^{\infty}(-1)^{i}(U(\Gamma, \vec{\alpha})-I)^{i}
$$

which is not necessarily convergent for all elements $X(\Gamma, \vec{\alpha})$. To compute this quantity we have swapped the order of two infinite summations and we performed first the summation along all the possible order of components of the multi-tensors in order to get the holonomy. We will repeat this procedure for other equations later on.

We will now define a new family of elements which has a well defined Lie group structure and for which the power-series holonomy is well defined. Consider the element of the algebra associated to the extended group of loops,

$$
\mathbf{F}(\gamma, M) \equiv \sum_{j=1}^{M} \frac{(-1)}{j}(I-\mathbf{X}(\gamma))^{j}
$$

and the following associated one parameter family of elements of the extended group of loops defined by,

$$
\mathbf{X}(\gamma, M, \lambda) \equiv \exp (\lambda \mathbf{F}(\gamma, M))
$$

The F's and $X$ 's satisfy the differential constraint. The $X$ 's are invertible simply by changing the sign of $\lambda$ and are elements of the group $\mathcal{D}_{o}$ of extended loops that satisfy the differential constraint base-pointed at $o$ and the $F$ 's are elements of its associated algebra $d_{o}$.

The motivation for this construction is to notice that in the limit,

$$
\lim _{M \rightarrow \infty} \mathbf{X}(\gamma, M, \lambda)=\mathbf{X}(\gamma)^{\lambda}
$$

the constructed element corresponds to the non-rational power $\lambda$ of the element $X(\gamma)$ of the group of loops. We will not, however, consider this limit in this subsection, since it yields a non-covariant holonomy.

The elements so introduced obviously form a group for fixed $\gamma$ and $M$ since,

$$
\mathbf{X}\left(\gamma, M, \lambda_{1}\right) \mathbf{X}\left(\gamma, M, \lambda_{2}\right)=\mathbf{X}\left(\gamma, M, \lambda_{1}+\lambda_{2}\right)
$$

Consider now the contraction of an element of the algebra $F$ with a connection. Such a contraction is well defined in terms of the holonomies along the loop $\gamma$,

$$
\mathbf{F}(\gamma, M) \cdot \mathbf{A}=\sum_{j=1}^{M} \frac{(-1)}{j}(\mathbf{I}-\mathbf{X} \cdot \mathbf{A})^{j}=\sum_{j=1}^{M} \frac{(-1)}{j}(\mathbf{I}-\mathbf{U})^{j} .
$$

and is gauge covariant since it is expressed as a finite sum of usual holonomies along ordinary loops.

The exponential of the contraction is also well defined in spite of the fact that it involves an infinite summation since the radius of convergence of the series expression of the exponential is infinite. The above expression is gauge covariant and is equal to the contraction of a connection with the exponential of the element of the algebra,

$$
\exp (\lambda \mathbf{F}(\gamma, M) \cdot \mathbf{A})=(\exp (\lambda \mathbf{F}(\gamma, M))) \cdot \mathbf{A}=\mathbf{X}(\gamma, M, \lambda) \cdot \mathbf{A}
$$

We have therefore explicitly constructed a family of elements of the group of loops $X(\gamma, M, \lambda)$ such that their powerseries holonomy is well defined. Since all expressions only involve combinations usual holonomies along ordinary loops they are gauge covariant under small and large gauge transformations.

The above family is a one-dimensional Lie group. One can construct an infinite dimensional Lie group in a straightforward manner, by considering the algebra generated by elements $\mathbf{F}(\gamma, M)$ for different loops $\gamma$. Any commutator $\left[\mathbf{F}\left(\gamma_{i}, M\right), \mathbf{F}\left(\gamma_{j}, M\right)\right]$ belongs to $d_{o}$ and has a well-defined contraction with a connection.

As we mentioned at the beginning, one can recover ordinary loops by considering the limit $M \rightarrow \infty$. Unfortunately, in that case it is not true that the power-series holonomy is well defined. If one considers expression (57) for that case one has a series that does not converge for all values of $\mathbf{U}$. For instance, for $S U(2)$ one could consider $\mathbf{U}=-\mathbf{I}$ and the series would not converge. All this is in agreement with the results of reference [26]. 


\section{An analytic extension of the power-series holonomy}

In this subsection we will define a different notion of holonomy, which can be viewed as an "analytic extension" of the power-series holonomy. The construction can be summarized as follows. We will fist introduce a family of extended loops satisfying the differential constraint, each labelled by a loop and a real number, $\mathbf{X}(\gamma, a)$ such that for a particular value of $a$ we get the usual multitensor $\mathbf{X}(\gamma)$. We will then consider the power-series holonomy built with these elements and will show that for certain range of $a$ it is well defined. The range will not include the value for which we recover the ordinary multi-tensors. We will then assign a holonomy for the forbidden range through an analytic extension. This will provide a notion of holonomy associated with a family of extended loops in $\mathcal{D}_{o}$ (including ordinary loops) that will coincide with the usual power-series holonomy only for a certain subset of elements.

Given a simply-traversed loop $\gamma$ and a real number $a$ consider the elements $\mathbf{X}(a, \gamma) \in \mathcal{D}_{o}$ defined by,

$$
\mathbf{X}(a, \gamma)=(1-a) \mathbf{I}+a \mathbf{X}(\gamma) .
$$

We now compute the power-series holonomy for such an element for a $U(1)$ connection?.

$$
U(\mathbf{X}(a, \gamma))=\mathbf{X}(a, \gamma) \cdot \mathbf{A}=(1-a)+a U(\gamma)
$$

Since $|U|=1$ then $0<|U(\mathbf{X}(a, \gamma))-1|<2 a$. Then we can construct an element of the algebra $d_{o}$ defined by

$$
\mathbf{F}(a, \gamma)=\sum_{i=1}^{\infty} \frac{(-1)}{i}(1-\mathbf{X}(a, \gamma))^{i}
$$

whose contraction with a connection,

$$
\mathbf{F}(a, \gamma) \cdot \mathbf{A}=\sum_{i=1}^{\infty} \frac{(-1)}{i}(1-U(\mathbf{X}(a, \gamma)))^{i}
$$

is well-defined and gauge invariant for $a<1 / 2$ (notice that we have made a choice in the order in which we perform the two infinite summations involved).

We can now introduce a one parameter family of elements of $\mathcal{D}_{o}$ obtained by exponentiating the elements of $d_{o}$ we just introduced times a real number,

$$
\mathbf{X}(a, \gamma)^{\lambda}=\exp (\lambda \mathbf{F}(a, \gamma))
$$

whose power-series holonomy is well-defined and that can be thought of as the real powers of the elements $\mathbf{X}(a, \gamma)$.

Two things need to be noticed. On the one hand, as we mentioned above, loops are included as a particular case of this family $(a=1)$. For that case however, the power-series holonomy for a real power $\mathbf{X}(a, \gamma)^{\lambda}$ is ill defined. The second observation is that although the above elements are associated with loops, they do not provide a representation of the product of loops in the sense that,

$$
\mathbf{X}\left(a, \gamma_{1}\right) \times \mathbf{X}\left(a, \gamma_{2}\right) \neq \mathbf{X}\left(a, \gamma_{1} \circ \gamma_{2}\right) .
$$

If one wants to recover loops as a particular case, one needs to evaluate the above expressions for $a=1$ which is outside the radius of convergence of the power-series expansions we have considered for the holonomy. One can proceed by summing the series in the convergence region to obtain a function of $a$ and then analytically extending such function to $a=1$. For the $U(1)$ case this can be performed explicitly in a straightforward fashion. One first observes that in the domain of convergence of the series,

$$
\mathbf{F}(a, \gamma) \cdot \mathbf{A} \equiv \log |U|+i \operatorname{Arg}(U)
$$

where $\operatorname{Arg}(U)$ is the principal part of the argument of the complex number $U$. This last prescription for the logarithm that is fixed by the power series expansion we were considering.

\footnotetext{
${ }^{1}$ Because our main goal is to define the $\lambda$-th power of a loop with $\lambda$ real it suffices to consider a simply traversed loop, since a multiply-traversed loop is a particular case of the construction with $\lambda$ integer.

${ }^{2}$ The generalization to other gauge groups is straightforward, one just needs to consider a gauge invariant norm in the space of matrices of the representation of the given group and require that the matrices involved be appropriately close to the identity.
} 
Therefore, exponentiating the above result, we get,

$$
U\left(\mathbf{X}(a, \gamma)^{\lambda}\right)=|U(\mathbf{X}(a, \gamma))|^{\lambda} \exp (i \lambda \operatorname{Arg}(U(\mathbf{X}(a, \gamma)))) .
$$

This expression coincides with the power series expansions we introduced, in the interval $0<a<1 / 2$. We now can analytically continue it to $a=1$ and obtain as result for the real power of an ordinary loop,

$$
U\left(\mathbf{X}(\gamma)^{\lambda}\right)=|U(\mathbf{X}(\gamma))|^{\lambda} \exp (i \lambda \operatorname{Arg}(U(\mathbf{X}(\gamma)))) .
$$

From here one can define a set of extended loops for which their holonomy is well defined and that includes loops as a particular case. Simply consider all the elements obtained by exponentiation of linear combinations of $\mathbf{F}\left(a, \gamma_{i}\right)$ 's for all possible loops and their commutators. We have a consistent prescription to assign them holonomies given above. It may even be possible that the set considered above in the case $a=1$ spans the whole $\chi_{o}$ (the set of extended loops satisfying both the algebraic and differential constraint). This is true in the Abelian case, in the non-Abelian case the issue is more subtle.

The set we have just constructed can be especially useful to define "thickened out loops", a procedure we outline in the next subsection.

\section{Thickened out loops}

One of the main motivations to consider extended loops was that there exists a formal set of quite nontrivial solutions to the Wheeler-DeWitt equation in loop space that is ill defined if one considers it in the loop representation. By considering its generalization to extended loops and appropriately restricting the set of extended loops considered to those that arise from smooth Y's the wavefunctions are well defined.

Unfortunately the set of extended loops considered faced the difficulty of yielding non-gauge invariant powerseries holonomies. The attitude that some researchers suggested in the face of this problem is that probably the extended loops were "too big" a set and that a smaller generalization of loop space would be enough to regularize the wavefunctions. An intuitive notion that is usually mentioned in this context is that of "thickened out loops" or "framed loops", that is, loops with some additional structure that allows to soften the divergences that the wavefunctions suffer when evaluated on ordinary distributional loops.

In this subsection we will show how to implement a practical version of this notion taking advantage of the constructions we introduced in the previous section.

Broadly speaking a "thickened out loop" is a tubular structure composed by a two-parameter family of loops $\gamma\left(s_{1}, s_{2}\right)$. A naive way to use such a structure would be to simply superpose the multi-tangents,

$$
\mathbf{X}_{\text {thick }}(\gamma)=\int d^{2} s \mathbf{X}_{\text {thick }}\left(\gamma\left(s_{1}, s_{2}\right)\right)
$$

Unfortunately, such a construction is not very useful in practice. If one inserts such an expression into one of the wavefunctions considered, the calculation amounts to contracting it with an appropriate group of (distributional) propagators. Since the integrand contracted with the propagators is divergent, the integral is not able to remove this divergence. Basically what is happening is that one would like to "separate" each entry contracted with a propagator on different loops. That is not accomplished by the above construction.

One way that seems plausible to achieve that goal is to consider a superposition similar to the one considered above, but instead of doing it in terms of elements of the extended group, to do it with elements of the extended algebra. The result can be exponentiated and an element of the group can be generated. Concretely,

$$
\begin{aligned}
& \mathbf{F}_{\text {thick }}(\gamma)=\int d^{2} s \mathbf{F}_{\text {thick }}\left(\gamma\left(s_{1}, s_{2}\right)\right) \\
& \mathbf{X}_{\text {thick }}(\gamma)=\exp \left(\mathbf{F}_{\text {thick }}(\gamma)\right)
\end{aligned}
$$

Up to present it has not been checked that the above proposal suffices to yield well-defined expressions for all the wavefunctions involved in the Kauffman bracket/Jones polynomial construction. It works, however, for the self-linking number. Consider the expression for the self-linking number evaluated on $\mathbf{X}_{\text {thick }}(\gamma)$,

$$
\operatorname{Linking}\left(\mathbf{X}_{\text {thick }}\right)=\left(\exp \left(\mathbf{F}_{\text {thick }}(\gamma)\right)\right)^{\mu_{1} \mu_{2}} g_{\mu_{1} \mu_{2}} .
$$

If we now write out the expansion of the exponential we get, for the rank two term, 


$$
\operatorname{Linking}\left(\mathbf{X}_{\text {thick }}\right)=\left(\mathbf{F}_{\text {thick }}^{\mu_{1} \mu_{2}}(\gamma)+\mathbf{F}_{\text {thick }}^{\mu_{1}} \mathbf{F}_{\text {thick }}^{\mu_{2}}\right) g_{\mu_{1} \mu_{2}} \text {. }
$$

Now, the problematic term would be the first one since it could happen that both indices are evaluated on the same loop. However that term vanishes because $\mathbf{F}$ is an element of the algebra and therefore satisfies the homogeneous algebraic constraint (it is antisymmetric in $\mu_{1}, \mu_{2}$ whereas $g$ is symmetric). The other term has two separate thickened out loops and therefore it is naturally regularized.

A task for the future is to continue this analysis for the other coefficients of the knot polynomials and see if the prescription allows to define them without singularities. It will then have to be studied what is the action of the regularized Wheeler-DeWitt equation on the resulting states. Notice that the general form of the constraints in the extended representation is the same as the one one had in the full extended loops. We are just changing the domain of dependence from that of extended loops stemming from smooth $Y$ 's to the one we discussed above.

\section{CONCLUSIONS AND OUTLOOK}

We have seen how the use of the Ashtekar variables and the loop representation in quantum gravity has suggested several heuristic results concerning the space of states of quantum gravity. In particular, one is able to find solutions to all the constraints of the theory (formally speaking) that are related to Chern-Simons theory. These are the only solutions known at present to all the constraints of quantum gravity in any formalism. To put such results on a rigorous setting we need to regularize the theory and we have seen that the use of extended loops seems a powerful avenue to perform this task. In this context one can for the first time find well defined solutions that solve the constraints in a regularized setting.

The main obstacle at present to consider these results as definitive is the observation that the use of the full extended group as an arena may not preserve the gauge covariance of the theory. We have introduced a sub-sector of the extended space in the previous section that preserves gauge invariance and that appears as a natural "thickening out" regularization for loops. In terms of these objects it is not clear anymore if the states we have proposed will be appropriately regularized and if the action of the constraints will be well defined. These issues are the next step we need to address in the quest for a regularized theory of quantum gravity.

[1] T. Jacobson, L. Smolin, Nuc. Phys. B299, 295 (1988).

[2] C. Rovelli, L. Smolin, Phys. Rev. Lett. 61, 1155 (1988).

[3] B. Brügmann, R. Gambini, J. Pullin, Gen. Rel. Grav. 25, 1 (1993).

[4] C. Di Bartolo, R. Gambini, J. Griego, Commun. Math. Phys. 158, 217 (1993).

[5] A. Ashtekar, J. Lewandowski, D. Marolf, J. Mourão, T. Thiemann J. Math. Phys. (this volume).

[6] R. Gambini, A. Trias, Phys. Rev. D23, 553 (1981).

[7] C. Rovelli, L. Smolin, J. Math. Phys. (this volume).

[8] R. Gambini, A. Trias, Nucl. Phys. B278, 436 (1986).

[9] R. Loll, Class. Quan. Grav. 10, 1471 (1993).

[10] D. Marolf, Class. Quan. Grav. 10, 2625 (1993).

[11] A. Ashtekar, "Recent Mathematical Developments in Quantum General Relativity", Preprint, CGPG-94/9-4, grqc@xxx.lanl.gov:9411055.

[12] R. Gambini, J. Pullin, "Loops, knots, gauge theories and quantum gravity", Cambridge University Press (to appear).

[13] L. Smolin, in Proceedings of the XXII Gift International Seminar on Theoretical Physics, Quantum Gravity and Cosmology, June 1991, Catalonia, Spain, World Scientific, Singapore (1992).

[14] R. Gambini, Phys. Lett. B255, 180 (1991).

[15] R. Gambini, J. Pullin, in "Knots and quantum gravity", J. Baez editor, Oxford University Press (1993).

[16] B. Brü̈mann, J. Pullin, Nucl. Phys. 390, 399 (1993).

[17] C. Rovelli, L. Smolin, Nuc. Phys. B331, 80 (1990).

[18] R. Borissov, "Regularization of the Hamiltonian constraint and the closure of the constraint algebra", preprint grqc@xxx.lanl.gov:9411038 (1994).

[19] C. Calugareanu, Czech. J. Math. 11, 588 (1961).

[20] E. Guadagnini, M. Martellini, M. Mintchev, Nucl. Phys. B227, 111 (1989).

[21] E. Witten, Commun. Math. Phys 121, 351 (1989). Lett. 68, 431 (1992).

[22] C. Rovelli, L. Smolin, Phys. Rev. Lett. 72, 446 (1994). 
[23] H. Kodama, Phys. Rev. D42, 2548 (1990).

[24] L. Smolin, Mod. Phys. Lett. A4 1091 (1989).

[25] C. Di Bartolo, R. Gambini, J. Griego, Phys. Rev. D51, 502 (1995).

[26] T. Schilling, "Non-covariance of the extended holonomy: examples", preprint (1995).

[27] B. Brügmann, R. Gambini, J. Pullin, Phys. Rev. Lett. 68, 431 (1992).

[28] R. Giles, Phys. Rev. D24, 2160 (1981). 